STOMACH

\title{
Helicobacter pylori upregulates matrilysin (MMP-7) in epithelial cells in vivo and in vitro in a Cag dependent manner
}

\author{
J R Bebb, D P Letley, R J Thomas, F Aviles, H M Collins, S A Watson, N M Hand, A Zaitoun, \\ J C Atherton
}

Gut 2003;52:1408-1413

See end of article for authors' affiliations ......................

Correspondence to: Professor J C Atherton, Division of

Gastroenterology,

University Hospital

Nottingham NG12 2NH,

UK:

john.atherton@

nottingham.ac.uk

Accepted for publication 17 June 2003

\begin{abstract}
Background and aims: Matrix metalloproteinase-7 (MMP-7) is important in normal and pathological remodelling of epithelial-matrix interactions, and is upregulated in gastric cancer. Helicobacter pylori infection is the first stage in gastric carcinogenesis, and therefore our aim was to determine if $\mathrm{H}$ pylori upregulated gastric MMP-7 expression and if this was affected by strain virulence.

Methods: We took gastric biopsy specimens at endoscopy from $H$ pylori infected $(n=17)$ and uninfected $(n=18)$ patients and assessed MMP-7 expression by ELISA, real time polymerase chain reaction (PCR), and immunohistochemistry (concentrating on epithelial cells in the proliferative zone). We PCR typed $H$ pylori for cagE and vacA. We performed $\mathrm{H}$ pylori/cell line coculture studies with wild-type pathogenic and non-pathogenic $\mathrm{H}$ pylori strains and with $\mathrm{CagE}^{-}$and $\mathrm{VacA}^{-}$isogenic mutants.

Results: Gastric biopsy specimens from $H$ pylorit patients expressed higher levels of MMP-7 at the protein and mRNA levels in the antrum and corpus (for example, by ELISA: H pylori+ 0.182 OD units $v \mathrm{H}$ pylori$0.059 ; p=0.009$ antrum). Epithelial cells from $\mathrm{H}$ pylori+ patients stained more intensely for MMP-7 than those from uninfected patients, including in the proliferative zone containing pluripotent cells $(p<0.03$ antrum, $\mathrm{p}<0.04$ body). Upregulation of MMP-7 in epithelial cells was confirmed at the protein and mRNA levels by $H$ pylori/cell line coculture. These experiments also showed that MMP-7 upregulation was dependent on an intact $H$ pylori cag pathogenicity island but not on the vacuolating cytotoxin.

Conclusion: We speculate that increased expression of MMP-7 in $\mathrm{H}$ pylori gastritis may contribute to gastric carcinogenesis.
\end{abstract}

$\mathrm{T}$ he main cause of peptic ulceration and gastric MALT lymphoma is Helicobacter pylori, ${ }^{2}$ and $H$ pylori is the strongest risk factor for the development of distal gastric adenocarcinoma. $^{3}$ The outcome of $H$ pylori infection is dependent on host, ${ }^{4}$ environmental, ${ }^{5}$ and bacterial factors. ${ }^{6} 7$ Strains possessing the cag pathogenicity island, encoding a type IV bacterial protein secretion system, ${ }^{8-11}$ are more strongly associated with increased levels of inflammation and disease, ${ }^{6}$ as are those producing an active form of VacA, a pore forming toxin that induces cytoplasmic vacuolation in vitro. ${ }^{12-15}$

Matrix metalloproteinases (MMPs) are a family of diverse zinc dependent proteolytic enzymes that are important in maintenance and remodelling of interactions between epithelial cells and basement membrane. ${ }^{16}$ They have many substrates, including collagen and elastin, and play an important role in promoting invasion and metastasis of cancer cells. MMPs also cleave cell surface bound substrates ("sheddase" activity) such as membrane bound cytokines, cytokine receptors, and adhesion molecules, releasing soluble or inactive forms. MMP-7 (matrilysin) is the smallest member of the MMP family and part of the stromelysin subclass. It is upregulated in epithelial cells in gastric cancer. $^{17-21}$ MMP-7 degrades various matrix substrates, including proteoglycans, gelatin, and elastin, and cleaves from the cell surface non-matrix substrates, including Ecadherin, pro-tumour necrosis factor $\alpha$ (TNF- $\alpha)$, and Fas ligand. ${ }^{22-26}$ A growing body of evidence suggests that MMP-7 plays an early role in tumorigenesis in colorectal cancer. The MMP-7 null Min mouse exhibits much reduced colonic tumour formation, ${ }^{27}$ and MMP-7 transcripts are found in the tumour epithelium of over $90 \%$ of colonic adenomas in both human and mice models. ${ }^{27} 28$

Here we have examined if MMP-7 is upregulated in H pylori gastritis, as this is the first stage in the progression to gastric carcinoma. We show that $H$ pylori upregulates MMP-7 expression at the mRNA level, and that MMP-7 is upregulated in inflammatory and epithelial cells, and crucially in the latter in the proliferative zone containing pluripotent cells. We show that this upregulation is caused by pathogenic strains of $H$ pylori, and is dependent on the cag pathogenicity island, but not on the toxin, VacA.

\section{METHODS}

In vivo studies

Patients and biopsy samples

Patients were recruited from routine day case endoscopy lists performed at University Hospital, Nottingham. Patients were not included if they had taken a proton pump inhibitor, antibiotics, or bismuth containing compounds in the previous four weeks. The study had full ethical approval from the University Hospital Nottingham Ethics Committee. Details of patient demographics are shown in table 1. Gastric biopsy specimens were taken from the antrum and corpus, and

\footnotetext{
Abbreviations: MMPs, matrix metalloproteinases; MMP-7, matrix metalloproteinase-7 (matrilysin); FCS, fetal calf serum; PCR, polymerase chain reaction; GAPDH, glyceraldehyde-3-phosphate dehydrogenase; $\mathrm{Ct}$, threshold cycle; SDS-PAGE, sodium dodecyl sulphatepolyacrylamide gel electrophoresis; ELISA, enzyme linked immunosorbent assay; TNF- $\alpha$, tumour necrosis factor $\alpha$
} 


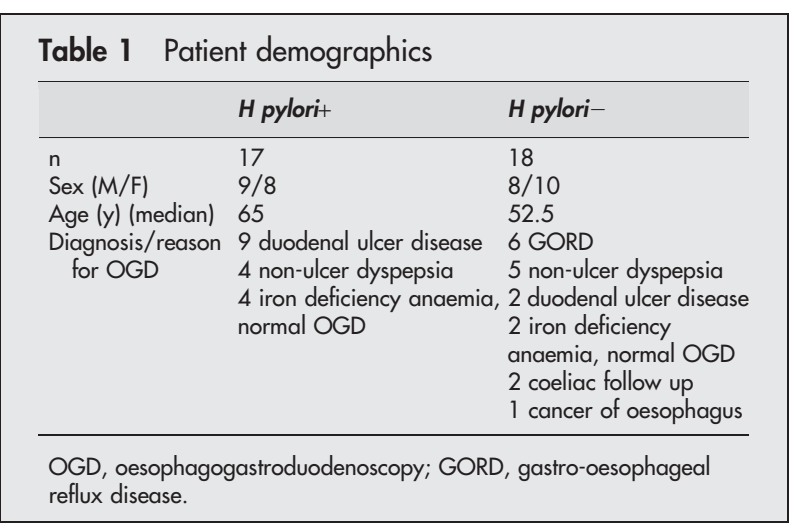

immediately snap frozen in liquid nitrogen for later RNA extraction and enzyme linked immunosorbent assay (ELISA) analysis. Adjacent biopsies were placed in formalin for routine histological analysis and for MMP-7 immunohistochemistry. Further adjacent biopsies from the antrum and body were used for the rapid urease test (Clotest; Ballard Medical Products, Draper, Utah, USA), and placed in Isosensitest media (Oxoid, Basingstoke, UK) supplemented with $5 \%$ fetal calf serum (FCS) for later $H$ pylori culture.

\section{MMP-7 ELISA}

The Quantikine human MMP-7 (total) ELISA kit (R\&D Systems, Abingdon, UK) was used to assess total MMP-7 in gastric antral biopsies from the first $10 \mathrm{H}$ pylori positive patients and $11 \mathrm{H}$ pylori negative patients. Biopsies, which had been snap frozen in liquid nitrogen, were homogenised using an Ultra-Turrax T50 homogeniser (Becton Dickenson, Oxford, UK) for 30 seconds on ice in $1 \mathrm{ml}$ of $20 \mathrm{mM}$ HEPES with added protease inhibitor cocktail (P8340, Sigma Aldrich, Dorset, UK). A standard curve was prepared using recombinant human MMP-7 according to the manufacturer's instructions. A spiked assay performed prior to running samples suggested there was no significant interference at the concentrations present in the biopsies. All ELISA scores were corrected according to the $\mathrm{A}_{280}$ of the initial biopsy homogenate.

\section{RNA extraction}

Total RNA was extracted from antral and corporal gastric biopsies (for 26 patients) using the Qiagen RNeasy kit (Crawley, UK) with additional DNAse digestion. Biopsies were initially homogenised as for the ELISA. RNA yields and purity were assessed using a GeneQuant pro RNA/DNA calculator (Biochrom; Amersham Pharmacia-Biotech, Chalfont St Giles, Bucks, UK).

\section{Real time semiquantitative PCR and analysis}

RNA was reverse transcribed using the Qiagen Omniscript Reverse Transcriptase kit with additional RNase inhibitor (10 units/reaction) and $1 \mu \mathrm{M}$ pdN6 salt random hexamer primer (Pharmacia, Amersham, UK). Reverse transcription was carried out at $37^{\circ} \mathrm{C}$ for one hour followed by five minutes at $93^{\circ} \mathrm{C}$ to inactivate the reverse transcriptase. A negative control (without Omniscript reverse transcriptase and random hexamer primer) was used for each sample. Real time polymerase chain reaction (PCR) was carried out using the QuantiTect SYBR Green PCR kit (Qiagen) using a GeneAmp 5700 Sequence Detection System (Applied Biosystems, California, USA) with 0.5 units of uracil-Nglycosylase per reaction and oligonucleotide primers specific for MMP-7 (5' AGCCAAACTCAAGGAGATGC3' and 5' ACTCCACATCTGGGCTTCTG3') and glyceraldehyde-3-phosphate dehydrogenase (GAPDH)
(5' GGTGAAGGTCGGAGTCAACGGA3' and 5' GAGGGATCTCGCTCCTGGAAGA3'). PCR conditions were as follows: $50^{\circ} \mathrm{C}$ for two minutes, $95^{\circ} \mathrm{C}$ for 15 minutes followed by 40 cycles of $94^{\circ} \mathrm{C}\left(15\right.$ seconds), $56^{\circ} \mathrm{C}$ (30 seconds), and $72^{\circ} \mathrm{C}$ (one minute). Relative expression of MMP-7 cDNA amplification, $\Delta \mathrm{Ct}(v$ GAPDH) was calculated using the formula $2^{\mathrm{Ct}}$ (GAPDH)-Ct (MMP-7) where $\mathrm{Ct}$ (threshold cycle) represents the fractional cycle number at which the fluorescence passes the fixed threshold. ${ }^{29}{ }^{30}$ The correct PCR product size was confirmed by electrophoresis on a $2 \%$ agarose gel.

\section{Immunohistochemistry}

Gastric biopsies were fixed in formol saline for 24 hours and subsequently processed and embedded in paraffin wax. Each biopsy was sectioned at $4 \mu \mathrm{M}$, with sufficient sections to permit one negative control, and stained with a specific monoclonal antibody to MMP-7 at a dilution of $1: 800$ (Oncogene Research Products, Cambridge, UK; cat No OM71). The sections were picked up on Dako ChemMate microscope slides (gap $75 \mu \mathrm{M}$ ) to allow immunoperoxidase staining on a Dako TechMate 500 Plus automated stainer (Dako, Ely, Cambs, UK). Staining was previously optimised (including pretreatment) for each antibody and a labelled streptavidin biotin technique employed to detect localisation of the antigens using diaminobenzine as the chromogen. An identical procedure (including pretreatment) was used for the negative control but the primary antibody was replaced with diluent only.

Briefly, the procedure required microwave antigen retrieval with $10 \mathrm{mM}$ citrate buffer $(\mathrm{pH} 6.0)$. All staining was performed at room temperature with an incubation of one hour for the primary antibody.

\section{Assessment of staining}

Two observers (AZ, an experienced histopathologist, and JB) assessed MMP-7 staining intensity. Initial observations suggested staining intensity differed between areas of biopsies, dependent on depth from the surface epithelium. We were particularly interested in staining in the proliferative zone, so we graded staining intensity here and in superficial and deep zones. Zones were defined by splitting the area between superficial cells and stromal cells into thirds. Staining intensity was graded 0 (no staining) to 4 (intense staining). Both observers were blinded to the $H$ pylori status of the individuals and they scored slides separately: 95\% concordance was reached on initial scoring. Discordant results were resolved by joint review, again with both scorers blind to the $H$ pylori status. Up to 200 inflammatory cells (where present) were also counted per field view (crossing all three zones), and the percentage expressing MMP-7 calculated.

$H$ pylori culture and PCR typing for cagE and vacA Gastric biopsies were plated out within one hour on $5 \%$ horse blood agar plates and left for five days in a $5 \% \mathrm{CO}_{2}$ incubator at $37^{\circ} \mathrm{C}$. Colonies of $\mathrm{H}$ pylori were identified by morphology, Gram stain, and urease testing, and clonally expanded before freezing at $-80^{\circ} \mathrm{C}$. Genomic DNA was extracted as previously described ${ }^{31}$ and strains were PCR typed for the presence of cagE, a reliable marker for the presence of the cag pathogenicity island (5' AAGGGTAAAGAAATGGGACTGAAT $3^{\prime}$ and $5^{\prime}$ GGAAGCGTGATAAAAGAGCAATGT $3^{\prime}$, conditions $95^{\circ} \mathrm{C}$ for two minutes, 35 cycles of $95^{\circ} \mathrm{C}$ for one minute, $56^{\circ} \mathrm{C}$ for two minutes, $72^{\circ} \mathrm{C}$ for four minutes, and $72^{\circ} \mathrm{C}$ for five minutes) and vacA (signal and mid region, as described previously ${ }^{32}{ }^{33}$ ). All PCR reactions were carried out on a Hybaid thermal cycler. 


\section{Definition of $H$ pylori status}

For the purposes of this study, patients were considered to be infected with $H$ pylori if culture was positive, or in the case of negative culture, if histology showed characteristic organisms and the Clotest rapid urease test was positive.

\section{In vitro studies}

\section{$H$ pylori strains}

The following strains were used for coculture experiments: 60190 (ATCC 49503, cagPaI+, vacA slml ${ }^{13}$ ), Tx30a (ATCC 51392, cagPaI-, vacA s2 $\left./ \mathrm{m}^{13}\right), 60190 \mathrm{CagE}^{-}$(cagE- insertion isogenic mutant of 60190, vacA sl/ml ), 60190 $\mathrm{VacA}^{-}$(cagPaI+, disrupted $\mathrm{vacA}^{-}$insertion mutant of $60190^{34}$ ). The mutant strain 60190 $\mathrm{CagE}^{-}$was derived from the cagE (picB) mutant 84-183:pMT3: $\mathrm{km}^{35}$ A $3.4 \mathrm{~kb}$ fragment containing the disrupted cagE gene was PCR amplified from 84-183:pTM3:km and cloned into pGEM T-Easy (obtained from Promega Corp., Southampton, UK) to create pRTl. The chromosomal cagE gene of strain 60190 was then replaced with the disrupted cagE gene by natural transformation with pRTl, followed by allelic exchange and kanamycin marker rescue. PCR analysis and Southern blotting confirmed replacement of cagE with the disrupted allele. We confirmed that the mutant strain was phenotypically null for the cag encoded type IV secretion system by showing that, compared with wild-type, it had a markedly reduced ability to stimulate epithelial cell lines to express interleukin 8 and that it did not induce CagA phosphorylation in epithelial cells (data not shown). The mutant strain $60190 \mathrm{VacA}^{-}$has been described previously. ${ }^{34}$

\section{Bacterial coculture}

H pylori strains 60190, Tx30a, and $\mathrm{CagE}^{-}$and $\mathrm{VacA}^{-}$isogenic mutants of $60190^{13}{ }^{34}$ were grown on $5 \%$ horse blood agar plates (Oxoid) in a $5 \% \mathrm{CO}_{2}$ incubator at $37^{\circ} \mathrm{C}$. HT29 cells (a human colonic adenocarcinoma cell line, obtained from the European Tissue Culture collection) were maintained in Dulbecco's modified Eagle's medium supplemented with 5\% FCS. For coculture, 48 hour plates of $H$ pylori were added to subconfluent HT29 (in the absence of FCS) in $75 \mathrm{~cm}^{2}$ tissue culture flasks such that the final viable bacteria:epithelial cell ratio was approximately 100:1. Tissue culture flasks were placed in a $5 \% \quad \mathrm{CO}_{2}$ incubator at $37^{\circ} \mathrm{C}$ for 24 hours. Supernatants were then aspirated and used for immunoblots and zymography, and cell pellets used for RNA extraction.

\section{Immunoblot and zymographic analysis of supernatants}

Equal volumes of untreated (media) and bacterial treated epithelial cell supernatants were analysed for the presence of MMP-7 by immunoblot and casein zymography. For immunoblots, samples were mixed with sodium dodecyl sulphate (SDS) running buffer, boiled, and centrifuged before application to $12.5 \%$ SDS-polyacrylamide gel electrophoresis (PAGE). For blotting, SDS-PAGE gels were blotted for one hour onto PVDF membranes at $150 \mathrm{~mA}$, blocked in phosphate buffered saline-0.5\% Tween with 5\% skimmed milk powder (Oxoid) for one hour, and then incubated with primary antibody (monoclonal anti-MMP-7; Oncogene Research Products, Nottingham, UK) overnight, washed and incubated with peroxidase conjugated goat antimouse IgG antibodies(Sigma-Aldrich), washed, and then developed using ECL system (Amersham Pharmacia-Biotech). For casein zymography, samples were mixed with SDS sample buffer (Novex; Invitrogen, Paisley, UK), applied to a $4-16 \%$ blue casein zymogram gel (Invitrogen) and run using the manufacturer's recommended buffers. After running, the gels were placed in renaturing buffer and incubated overnight in developing buffer (both buffers from Invitrogen).
Areas of protease activity appeared as white bands on a blue background and were photographed using a digital camera.

RNA extraction and real time PCR analysis

RNA extraction, quantification, and real time PCR analysis was carried out in an identical manner to the in vivo studies.

\section{Statistical analysis}

All statistical analyses used the GraphPad Prism statistical package. Mann-Whitney $U$ tests were used to compare median scores for histological grading. T tests were used to compare $\Delta \mathrm{Ct}$ values for mRNA and for comparing ELISA values.

\section{RESULTS}

We first aimed to assess whether MMP-7 expression in the gastric mucosa was upregulated by $H$ pylori infection. To do this we performed a specific ELISA for total MMP-7 on gastric biopsy specimen homogenates from $H$ pylori infected patients and uninfected control patients. MMP-7 was expressed in infected and uninfected gastric mucosa, with a threefold increase observed in $H$ pylori infected patients (mean ELISA score (SEM): H pylorit $(\mathrm{n}=10) 0.182(0.045) v$ H pylori- $(\mathrm{n}=11) 0.059$ (0.016); $\mathrm{p}=0.009$, unpaired $t$ test $)$. To confirm these results and to assess whether $H$ pylori infection upregulated MMP-7 expression at the transcriptional level, we extracted total RNA from biopsy specimens, reverse transcribed, and quantified levels using real time PCR. Biopsies from $H$ pylori infected patients expressed higher levels of MMP-7 mRNA in both the antrum ( $\triangle \mathrm{Ct}$ (SEM): $H$ pylori+ 0.038 (0.01) $(\mathrm{n}=12)$ v $H$ pylori- $0.008 \quad(0.003)$ $(\mathrm{n}=13) ; \mathrm{p}=0.007, t$ test $)$ and corpus (H pylori+ 0.044 $(0.021) \quad(\mathrm{n}=12) \quad v \quad H$ pylori- $0.005 \quad(0.002) \quad(\mathrm{n}=13)$; $p=0.07)$. We had more samples for RNA analysis than for ELISAs because RNA extraction was performed first, and where this was unsuccessful the biopsy intended originally for ELISA was used.

Our principal interest in MMP-7 upregulation was in its implications for the pathogenesis of gastric adenocarcinoma. Thus we aimed firstly to assess whether upregulation occurred in epithelial cells and secondly whether it occurred in the proliferative zone, which contains gastric stem cells. To do this, we performed immunohistochemistry on biopsy specimens from the gastric antrum and corpus for MMP-7 using a specific anti-MMP-7 monoclonal antibody and assessed epithelial staining intensity in epithelial cells in the proliferative zone. Staining intensity scores were significantly higher in epithelial cells in the proliferative zone in gastric biopsy specimens from $H$ pylori infected patients than in those from uninfected patients (median scores (interquartile range): antrum $H$ pylori +3 (3-4), H pylori- 3 (2-3); corpus $H$ pylori+ $3(2-3.5), H$ pylori- $2(2-2.5))$, both in the antrum $(\mathrm{p}=0.03$ Mann-Whitney $U$ test $)$ and the corpus $(p=0.04)$ (fig $1 \mathrm{~A}, \mathrm{~B})$. This is illustrated clearly in fig 2 , with intense proliferative zone staining in the $H$ pylori infected patient (fig 2A) but not in the uninfected patient (fig 2B). There was a trend for increased intensity staining in superficial and deep zones for $H$ pylori infected patients but these did not reach statistical significance (data not shown). Inflammatory cell MMP-7 expression is also of potential importance as MMP-7 released from inflammatory cells may exert paracrine effects on neighbouring epithelial cells. Thus, next we assessed the proportion of inflammatory cells staining for MMP-7 in $H$ pylorit and $H$ pylori- biopsy specimens. In $H$ pylori+ biopsy specimens, most inflammatory cells stained positively for MMP-7 whereas in $H$ pyloribiopsy specimens most did not stain (mean (SEM) per cent inflammatory cells staining positively for MMP-7: antrum, 

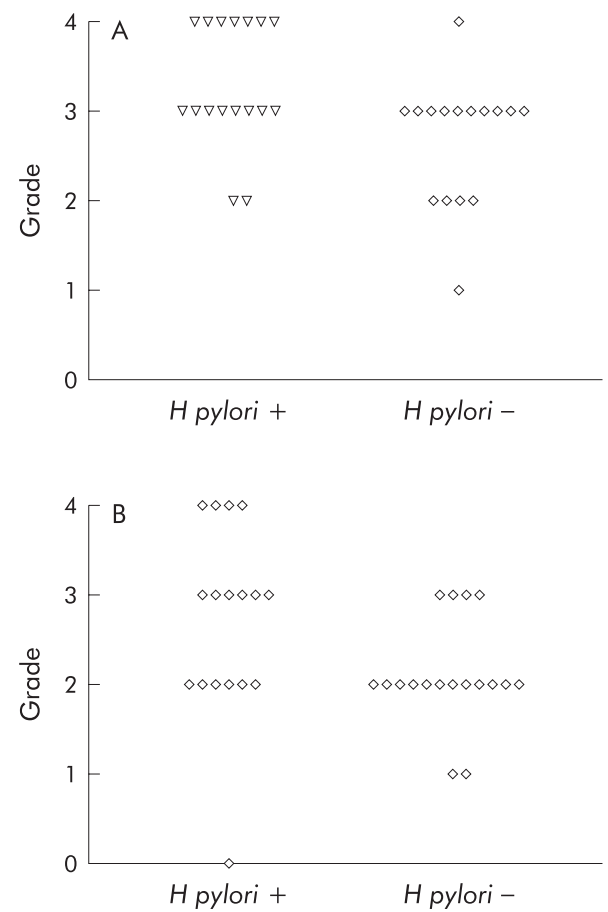

Figure 1 Dot plot showing epithelial cell staining grade $(0-4)$ for the proliferative zone in the antrum (A) and corpus (B) for Helicobacter pylorit and $\mathrm{H}$ pylori- patients. Grades were higher for $\mathrm{H}$ pylori infected patients in both the antrum ( $p=0.03$ Mann-Whitney $U$ test) and corpus $(p=0.04)$.

$H$ pylori+ 87.4 (2.8) v H pylori- 29.9 (4.1), p $<0.01 t$ test; corpus, $H$ pylorit 80.4 (2.7) v H pylori- 38.6 (4.4), $\mathrm{p}<0.01)$.

Because of the variation in level of MMP-7 expression between $H$ pylori+ biopsy specimens, especially with regard to epithelial cell expression, we next asked whether strains of differing virulence were associated with different levels of MMP-7 expression. To do this, we assessed cagE status and vacA type of $H$ pylori isolates cultured from gastric antral biopsy specimens and correlated these with MMP-7 expression. Unfortunately, only three strains were cagE negative, but nevertheless, by MMP-7 ELISA, cagE positive strains appeared to induce higher levels of MMP-7 expression than cagE negative strains (mean (SEM): cagE +0.218 (0.06), $\mathrm{n}=7 ;$ cagE -0.099 (0.019), $\mathrm{n}=3 ; \mathrm{p}=0.25$, unpaired $t$ test $)$. No obvious differences were demonstrated between cagE+ and cagE- strains by real time PCR or immunohistochemistry. $v a c A$ genotyping of strains revealed $2 \mathrm{sl} / \mathrm{ml}, 6 \mathrm{sl} / \mathrm{m} 2,1 \mathrm{~s} 2 /$ $\mathrm{m} 2$, and $1 \mathrm{~s} 2 / \mathrm{ml}$ (for ELISA and real time PCR). Not surprisingly, given the small numbers, no significant differences in MMP-7 expression could be demonstrated.

To define precisely the role of bacterial virulence factors in induction of MMP-7 expression and to confirm the effects of H pylori on MMP-7 expression by epithelial cells, we next performed a series of coculture experiments using a panel of $H$ pylori isogenic mutant strains and the epithelial cell line HT29. We selected the HT29 cell line because it is known to express MMP-7 in response to appropriate stimuli. ${ }^{36}$ Firstly, we analysed the effect of a pathogenic and a non-pathogenic H pylori strain on MMP-7 expression. Immunoblot analysis of HT29 supernatants after $H$ pylori coculture showed an immunoreactive $29 \mathrm{kDa}$ band (the predicted size for proMMP-7) after coculture with the cag positive vacuolating strain 60190 but no band following coculture with the cag negative non-vacuolating strain Tx30a, or for control cells without bacterial coculture. Next we defined the contribution of the vacuolating cytotoxin VacA and the cag PAI encoded
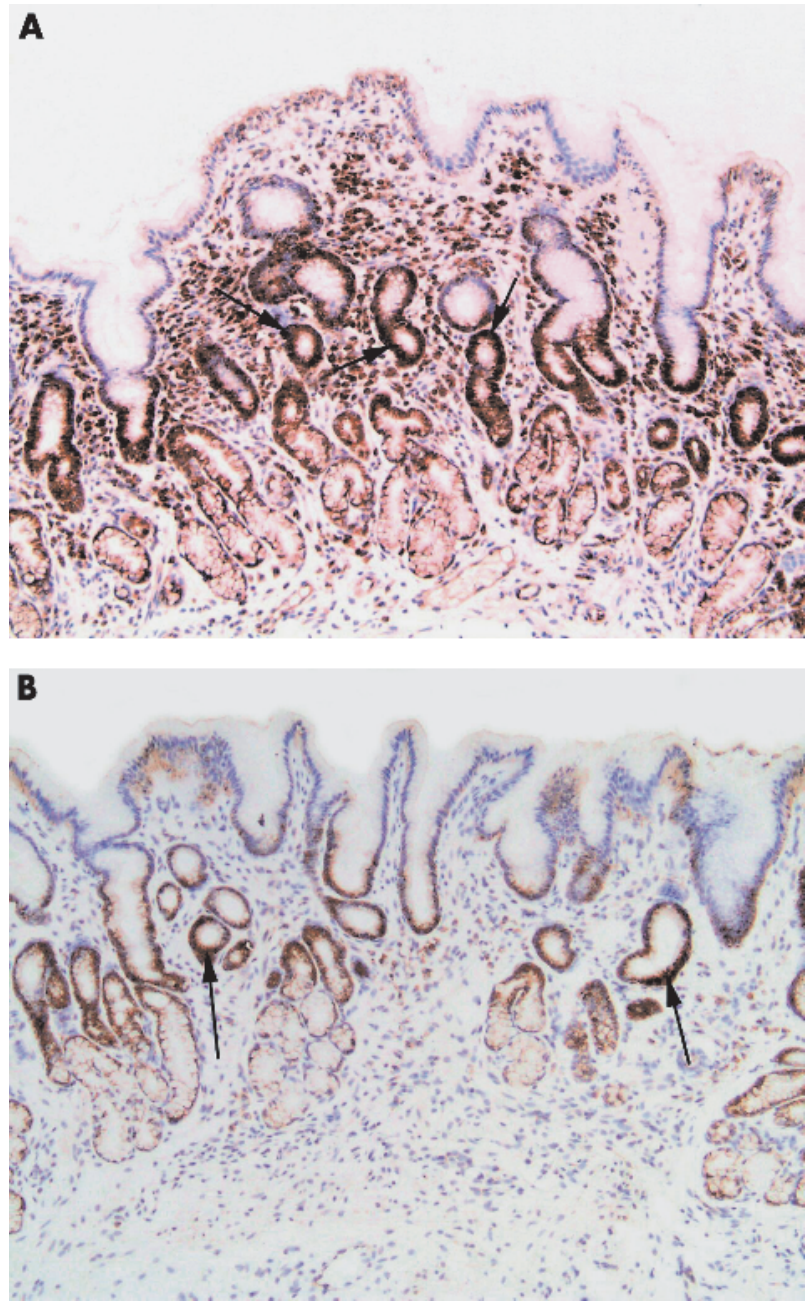

Figure 2 Antral biopsy stained for matrix metalloproteinase-7 (MMP-7) in a Helicobacter pylori infected patient (A) and uninfected patient (B). Note the intense staining for MMP-7 both within epithelial cells (particularly within the proliferative zone, arrows) and inflammatory infiltrate in (A).

type IV secretion system by comparing the effect on MMP-7 expression by HT29 cells of wild-type 60190 with the effects of its VacA- isogenic mutant (does not express full length or truncated VacA) and its CagE - isogenic mutant (does not express a functional cag encoded type IV secretion system). We showed that the VacA - mutant had identical effects to the parent strain, indicating that VacA was not necessary for the effect. In contrast, coculture with the $\mathrm{CagE}^{-}$mutant induced no MMP-7 expression, indicating that the effect was dependent on the cag PAI encoded type IV secretion system (fig 3A). To confirm these results, and to show that the $29 \mathrm{kDa}$ proMMP-7 band had MMP-7 activity, we performed casein zymography with the same samples. Zymograms demonstrated caseinolytic activity at the same protein size, and increased activity was noted for strain 60190 and its VacA $^{-}$isogenic mutant compared with the two strains lacking an intact cag pathogenicity island (Tx30a and $60190 \mathrm{CagE}-$ ) (fig 3B). Finally, to further confirm our results and to assess whether MMP-7 was upregulated at the level of transcription (as in our in vivo studies) we performed real time PCR on RNA preparations from cocultured HT29 cells. As expected, highest levels of MMP-7 mRNA expression were observed after coculture with the strains possessing an intact cag PAI, 60190, and 60190VacA ${ }^{-}$(fig 4). 



Figure 3 (A) Representative matrix metalloproteinase-7 (MMP-7) immunoblot of HT29 cell supernatants after coculture with Helicobacter pylori strains 60190, Tx30a, and the CagE- and VacA- isogenic mutants of 60190 . A band of approximately $29 \mathrm{kDa}$ is visualised for coculture with 60190 and $60190 \mathrm{VacA}-$. This corresponds to the predicted size for pro-MMP-7. No bands are seen with untreated cells, non-pathogenic strain Tx30a, and the CagE- isogenic mutant of 60190 . Equal volumes of supernatant were loaded onto sodium dodecyl sulphate-polyacrylamide gel electrophoresis $(n=4)$. (B) Representative casein zymogram demonstrating increased intensity bands with 60190 and $60190 \mathrm{VacA}-$. This $29 \mathrm{kDa}$ band corresponds to the caseinolytic activity of pro-MMP-7.

\section{DISCUSSION}

We have demonstrated that $H$ pylori infection causes upregulation of MMP-7 in epithelial cells, in vivo and in vitro, and that this is dependent on an intact cag pathogenicity island. MMP-7 is an important metalloproteinase enzyme that is upregulated in gastric cancer. It promotes tissue invasion and metastasis in various cancers through degradation of extracellular matrix. Interestingly, it has also recently been shown to have potential pro-oncogenic effects through its "sheddase" activity which may predispose to malignant transformation. Recently described substrates of MMP-7 sheddase activity include E-cadherin, Fas ligand, and pro-TNF- $\alpha$. E-cadherin is an important cell adhesion molecule which forms a key part of the adherens junctions between epithelial cells. ${ }^{37}$ Changes in E-cadherin at the germline and somatic levels are associated with both intestinal and diffuse gastric cancer. ${ }^{38-40}$ Fas ligand has an important role in apoptosis, and one of the mechanisms by which H pylori is thought to predispose to gastric carcinogenesis is through disruption of the balance between cell proliferation and apoptosis. ${ }^{41-43}$ TNF- $\alpha$ is a proinflammatory cytokine that is induced in vivo by $H$ pylori ${ }^{44}$ Induction of a chronic inflammatory state predisposes to gastric atrophy and precancerous changes in the gastric epithelium. ${ }^{41}$ Activation or alteration of any of these important oncogenic or inflammatory molecules by $H$ pylori potentially could be important in gastric carcinogenesis.

Our work using isogenic mutants clearly demonstrates a role for an intact cag pathogenicity island in the upregulation of MMP-7. The mechanism of upregulation of MMP-7 gene expression is likely to be complex as the promoter region is susceptible to a number of controls; ${ }^{45}$ MMP-7 transcription has been shown to be dependent on various signalling pathways, including $\beta$-catenin signalling ${ }^{36}{ }^{46}$ and activation of the PEA3 member of the Ets transcription factors. ${ }^{47}$ Translocation and phosphorylation of CagA stimulates MAP kinases and the cag encoded type IV secretion system itself stimulates nuclear factor $\mathrm{\kappa B}$ activation and interleukin 8 transcription. ${ }^{48} 49$ Further work is needed to define which pathways are responsible for $H$ pylori cag mediated MMP-7 induction. Most of our patient strains were cagE+ by PCR analysis, yet there was considerable heterogeneity in MMP-7

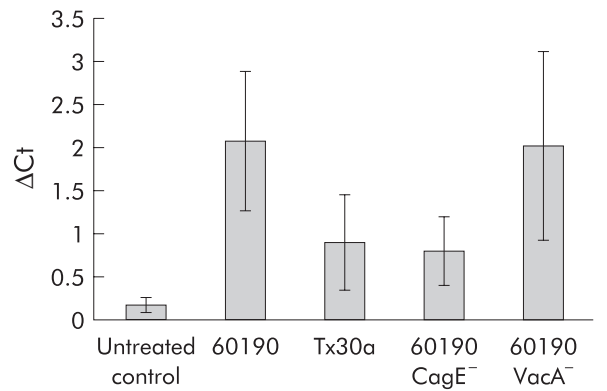

Figure 4 Matrix metalloproteinase-7 (MMP-7) mRNA values (mean (SEM) threshold cycle ( $\Delta \mathrm{Ct}$ ), corrected for glyceraldehyde-3-phosphate dehydrogenase) obtained by real time polymerase chain reaction for HT29 cell lysates after coculture with four Helicobacter pylori strains. Untreated control was uninnoculated media added to HT29 cells $(n=3-5$ per experiment). Interstrain differences did not reach conventional statistical significance but a similar trend to the protein data was observed.

expression in biopsies, and so clearly factors other than cag status may be important in MMP-7 upregulation, and the in vivo situation may be different from that in vitro.

Several groups have examined MMP-7 expression in gastric cancer and reported conflictingly on expression of MMP-7 in normal (non-malignant) tissue. ${ }^{17-20}$ Using a well defined monoclonal antibody raised against recombinant human MMP-7, we demonstrated clearly that MMP-7 is expressed in inflamed and to a lesser extent in non-inflamed non-malignant gastric mucosa, and we confirmed these findings by mRNA analysis. Importantly, for a potential mechanistic role in carcinogenesis, we have also shown that major differences exist in the proliferative zone between $H$ pylori infected and uninfected patients. Effects on cells within this pluripotent area are likely to be necessary for carcinogenesis.

There is considerable evidence to support a role for MMP-7 in the very early stages of colorectal cancer. ${ }^{27}{ }^{50}$ Induction of MMP-7 in H pylori induced gastritis, the initial stage in the proposed pathway to gastric adenocarcinoma, ${ }^{41}$ may be of pathogenic importance. Future strategies for treatment, and prevention, of gastric cancer may include use of inhibitors of metalloproteinase enzymes. Understanding what stimulates these oncogenic proinflammatory proteins adds to our current knowledge in this field. H pylori mediated stimulation of MMP-7 is a potentially important carcinogenic effect of the bacterium.

\section{ACKNOWLEDGEMENTS}

J Bebb was supported by fellowships from the Wellcome Trust (Entry Level) and the Medical Research Council (Clinical Training Fellowship). John Atherton holds a senior clinical fellowship from the Medical Research Council.

\section{Authors' affiliations}

J R Bebb, D P Letley, R J Thomas, F Aviles, J C Atherton, Division of Gastroenterology and Institute of Infections, Immunity, and Inflammation, University Hospital, Nottingham, UK

H M Collins, S A Watson, Academic Unit of Cancer Studies, University Hospital, Nottingham, UK

N M Hand, A Zaitoun, Histopathology Department, University Hospital, Nottingham, UK

\section{REFERENCES}

1 Nomura A, Stemmermann GN, Chyou PH, et al. Helicobacter pylori infection and gastric carcinoma among Japanese Americans in Hawaii. N Engl J Med 1991;325:1132-6.

2 Parsonnet J, Friedman GD, Vandersteen DP, et al. Helicobacter pylori infection and the risk of gastric carcinoma. NEngl J Med 1991;325:1127-31. 
3 Nomura A, Stemmermann GN, Chyou PH, et al. Helicobacter pylori infection and the risk for duodenal and gastric ulceration. Ann Intern Med 1994; 120:977-81.

4 El-Omar EM, Carrington M, Chow WH, et al. Interleukin-1 polymorphisms associated with increased risk of gastric cancer. Nature 2000;404:398-402.

5 Martin DF, Montgomery E, Dobek AS, et al. Campylobacter pylori, NSAIDS, and smoking: risk factors for peptic ulcer disease. Am J Gastroenterol 1989;84:1268-72.

6 Atherton JC. H. pylori virulence factors. Br Med Bull 1998;54:105-20.

7 Blaser MJ, Berg DE. Helicobacter pylori genetic diversity and risk of human disease. J Clin Invest 2001;107:767-73.

8 Asahi M, Azuma T, Ito S, et al. Helicobacter pylori CagA protein can be tyrosine phosphorylated in gastric epithelial cells. J Exp Med 2000;191:593-602.

9 Odenbreit S, Puls J, Sedlmaier B, et al. Translocation of Helicobacter pylori CagA into gastric epithelial cells by type IV secretion. Science 2000;287: 1497-500.

10 Segal ED, Cha J, Lo J, et al. Altered states: involvement of phosphorylated CagA in the induction of host cellular growth changes by Helicobacter pylori. Proc Natl Acad Sci U S A 1999;96:14559-64.

11 Stein M, Rappuoli R, Covacci A. Tyrosine phosphorylation of the Helicobacter pylori CagA antigen after cag-driven host cell translocation. Proc Natl Acad Sci U S A 2000;97:1263-8.

12 Atherton JC. The clinical relevance of strain types of Helicobacter pylori. Gut 1997;40:701-3.

13 Leunk RD, Johnson PT, David BC, et al. Cytotoxic activity in broth-culture filtrates of Campylobacter pylori. J Med Microbiol 1988;26:93-9

14 Papini E, de Bernard M, Milia E, et al. Cellular vacuoles induced by Helicobacter pylori originate from late endosomal compartments. Proc Natl Acad Sci U S A 1994;91:9720-4.

15 Szabo I, Brutsche S, Tombola F, et al. Formation of anion-selective channels in the cell plasma membrane by the toxin VacA of Helicobacter pylori is required for its biological activity. EMBO J 1999;18:5517-27.

16 McCawley LJ, Matrisian LM. Matrix metalloproteinases: they're not just for matrix anymore! Cur Opin Cell Biol 2001;13:534-40.

17 Adachi $Y$, Itoh $F$, Yamamoto $H$, et al. Matrix metalloproteinase matrilysin (MMP-7) participates in the progression of human gastric and esophageal cancers. Int J Oncol 1998;13:1031-5.

18 Honda M, Mori M, Ueo $\mathrm{H}$, et al. Matrix metalloproteinase-7 expression in gastric carcinoma. Gut 1996;39:444-8

19 McDonnell S, Coffey M, Navre RJ jr, et al. Expression and localization of the matrix metalloproteinase pump-1 (MMP-7) in human gastric and colon carcinomas. Mol Carcinog 1991;4:527-33.

20 Senota A, Itoh F, Yamamoto $H$, et al. Relation of matrilysin messenger RNA expression with invasive activity in human gastric cancer. Clin Exp Metastasis 1998;16:313-21.

21 Yamashita K, Azumano I, Mai M, et al. Expression and tissue localization of matrix metalloproteinase 7 (matrilysin) in human gastric carcinomas. Implications for vessel invasion and metastasis. Int J Cancer 1998;79:187-94.

22 Noe V, Fingleton B, Jacobs K, et al. Release of an invasion promoter Ecadherin fragment by matrilysin and stromelysin-1. J Cell Sci 2001;114:111-18.

23 Powell WC, Fingleton B, Wilson $\mathrm{CL}$, et al. The metalloproteinase matrilysin proteolytically generates active soluble Fas ligand and potentiates epithelial cell apoptosis. Curr Biol 1999:9:1441-7.

24 Lochter A, Galosy S, Muschler J, et al. Matrix metalloproteinase stromelysin-1 triggers a cascade of molecular alterations that leads to stable epithelial-tomesenchymal conversion and a premalignant phenotype in mammary epithelial cells. J Cell Biol 1997; 139:1861-72.

25 Haro H, Crawford HC, Fingleton B, et al. Matrix metalloproteinase-7dependent release of tumor necrosis factor-alpha in a model of herniated disc resorption. J Clin Invest 2000;105: 143-50.

26 Mitsiades N, Yu WH, Poulaki V, et al. Matrix metalloproteinase-7-mediated cleavage of Fas ligand protects tumor cells from chemotherapeutic drug cytotoxicity. Cancer Res 2001;61:577-81.
27 Wilson $\mathrm{CL}$, Heppner $\mathrm{KJ}$, Labosky PA, et al. Intestinal tumorigenesis is suppressed in mice lacking the metalloproteinase matrilysin. Proc Natl Acad Sci U S A 1997;94:1402-7.

28 Takeuchi N, Ichikawa Y, Ishikawa T, et al. Matrilysin gene expression in sporadic and familial colorectal adenomas. Mol Carcinog 1997;19:225-9.

29 Anonymous. User Bulletin\#2, ABI PRISM 7700 Sequence detection systems. Warrington: PE Applied Biosystems, 1997; December 11:1-36.

$30 \mathrm{Pfaffl} \mathrm{MW}$. A new mathematical model for relative quantification in real-time RT-PCR. Nucleic Acids Res 2001 ;29:2002-7.

31 Atherton JC. Molecular methods for detecting ulcerogenic strains of $\mathrm{H}$. pylori. In: Clayton CLMH, ed. Methods in molecular medicine, Helicobacter pylori protocols. Totawa: Humana Press, 1997:133-43.

32 Atherton JC, Cao P, Peek RM jr, et al. Mosaicism in vacuolating cytotoxin alleles of Helicobacter pylori. Association of specific vacA types with cytotoxin production and peptic ulceration. J Biol Chem 1995;270:17771-7.

33 Atherton JC, Cover TL, Twells RJ, et al. Simple and accurate PCR-based system for typing vacuolating cytotoxin alleles of Helicobacter pylori. J Clin Microbiol 1999;37:2979-82

34 Bebb JR, Letley DP, Rhead JL, et al. Helicobacter pylori supernatants cause epithelial cytoskeletal disruption that is bacterial strain and epithelial cell line dependent but not toxin VacA dependent. Infect Immun 2003;71:3623-7.

35 Tummuru MK, Sharma SA, Blaser MJ. Helicobacter pylori picB, a homologue of the Bordetella pertussis toxin secretion protein, is required for induction of IL-8 in gastric epithelial cells. Mol Microbiol 1995; 18:867-76.

36 Brabletz T, Jung A, Dag S, et al. beta-catenin regulates the expression of the matrix metalloproteinase-7 in human colorectal cancer. Am J Pathol 1999;155:1033-8.

37 Gumbiner BM. Cell adhesion: the molecular basis of tissue architecture and morphogenesis. Cell 1996;84:345-57.

38 Guilford P, Hopkins J, Harraway J, et al. E-cadherin germline mutations in familial gastric cancer. Nature 1998;392:402-5.

39 Becker KF, Atkinson MJ, Reich U, et al. E-cadherin gene mutations provide clues to diffuse type gastric carcinomas. Cancer Res 1994;54:3845-52.

40 Jawhari A, Jordan S, Poole S, et al. Abnormal immunoreactivity of the Ecadherin-catenin complex in gastric carcinoma: relationship with patient survival. Gastroenterology 1997; 112:46-54.

41 Correa P. Helicobacter pylori and gastric carcinogenesis. Am J Surg Pathol 1995; 19:S37-43.

42 Rudi J, Kuck D, Strand S, et al. Involvement of the CD95 (APO-1/Fas) receptor and ligand system in Helicobacter pylori-induced gastric epithelial apoptosis. $J$ Clin Invest 1998;102:1506-14.

43 Wagner S, Beil W, Westermann J, et al. Regulation of gastric epithelial cell growth by Helicobacter pylori: offdence for a major role of apoptosis. Gastroenterology 1997;113:1836-47.

44 Crabtree JE, Shallcross TM, Heatley RV, et al. Mucosal tumour necrosis factor alpha and interleukin-6 in patients with Helicobacter pylori associated gastritis. Gut 1991;32:1473-7.

45 Gaire M, Magbanua Z, McDonnell S, et al. Structure and expression of the human gene for the matrix metalloproteinase matrilysin. J Biol Chem 1994; 269:2032-40.

46 Crawford HC, Fingleton BM, Rudolph-Owen LA, et al. The metalloproteinase matrilysin is a target of beta-catenin transactivation in intestinal tumors. Oncogene 1999; 18:2883-91.

47 Crawford HC, Fingleton B, Gustavson MD, et al. The PEA3 subfamily of Ets transcription factors synergizes with beta-catenin-LEF-1 to activate matrilysin transcription in intestinal tumors. Mol Cell Biol 2001;21:1370-83.

48 Meyer-ter-Vehn T, Covacci A, Kist M, et al. Helicobacter pylori activates mitogen-activated protein kinase cascades and induces expression of the proto-oncogenes c-fos and c-jun. J Biol Chem 2000;275: 16064-72.

49 Naumann M, Wessler S, Bartsch C, et al. Activation of activator protein 1 and stress response kinases in epithelial cells colonized by Helicobacter pylori encoding the cag pathogenicity island. J Biol Chem 1999;274:31655-62.

50 Witty JP, McDonnell S, Newell KJ, et al. Modulation of matrilysin levels in colon carcinoma cell lines affects tumorigenicity in vivo. Cancer Res 1994:54:4805-12. 